\title{
Contrast-enhanced ultrasound liver reporting and data system for hepatocellular carcinoma diagnosis
}

\author{
Alexander M. Vezeridis', Yuko Kono² \\ IInterventional Radiology, Stanford University Medical Center, Stanford, CA 94305, USA. \\ ²Department of Radiology, Department of Medicine, University of California, San Diego, CA 92103, USA.
}

Correspondence to: Prof. Yuko Kono, Department of Radiology, Department of Medicine, University of California, 200 W Arbor Dr. MC 8413, San Diego, CA 92103, USA. E-mail: ykono@health.ucsd.edu

How to cite this article: Vezeridis AM, Kono Y. Contrast-enhanced ultrasound liver reporting and data system for hepatocellular carcinoma diagnosis. Hepatoma Res 2020;6:53. http://dx.doi.org/10.20517/2394-5079.2020.36

Received: 14 Apr 2020 First Decision: 25 May 2020 Revised: 16 Jul 2020 Accepted: 21 Jul 2020 Published: 21 Aug 2020

Academic Editor: Guang-Wen Cao Copy Editor: Cai-Hong Wang Production Editor: Jing Yu

\begin{abstract}
Contrast-enhanced ultrasound (CEUS) is a powerful imaging modality for the diagnosis of focal liver lesions, including hepatocellular carcinoma (HCC). The American College of Radiology Contrast-Enhanced Ultrasound Liver Reporting and Data System (CEUS LI-RADS ${ }^{\circledR}$ ) was created as a standardized reporting system to facilitate consistent and high-quality technique, interpretation, reporting, and data collection for CEUS diagnosis of HCC. This article describes the history and background of CEUS LI-RADS ${ }^{\circledR}$, its major concepts and algorithm, and the differences between CEUS LI-RADS ${ }^{\circledR}$ and CT/MRI LI-RADS ${ }^{\circledR}$.
\end{abstract}

Keywords: Contrast-enhanced ultrasound, LI-RADS, hepatocellular carcinoma, diagnosis

\section{INTRODUCTION}

Hepatocellular carcinoma (HCC) can be diagnosed non-invasively by imaging without histological confirmation as endorsed by major liver society guidelines ${ }^{[1,2]}$. Contrast-enhanced computed tomography (CT) and magnetic resonance imaging (MRI) have been used for HCC diagnosis, with contrastenhanced ultrasound (CEUS) recognized as an alternative imaging modality ${ }^{[3]}$. Indeed, focal liver lesion characterization is the most widely recognized and used application for CEUS ${ }^{[4]}$.

CEUS is an advanced form of ultrasound imaging utilizing tiny microbubble contrast agents, with diameters similar to that of red blood cells ${ }^{[5]}$. After intravenous injection, the microbubbles stay in the 
bloodstream for minutes, allowing ultrasound imaging machines with specialized contrast-specific modes to visualize differences in the vascularity of tissues of interest ${ }^{[6]}$. The microbubbles resonate and exhibit harmonic signals under very weak ultrasound exposure while the background tissues exhibit only a linear response to ultrasound signals ${ }^{[7]}$. Contrast-specific imaging utilizes this unique property of microbubble contrast agents, and cancels the linear tissue signal, resulting in a contrast-enhanced microbubble-only image ${ }^{[8]}$. CEUS is highly sensitive owing to the intrinsically high contrast of microbubbles ${ }^{[7]}$.

Microbubble contrast agents demonstrate multiple contrast phases in the liver after they are injected intravenously, progressing from arterial phase ( 10-20 s to 30-45 s after injection) to portal venous phase $(\sim 30-45 \mathrm{~s} \text { to } 2 \mathrm{~min} \text { after injection) to late phase ( } \sim 2 \mathrm{~min} \text { to } 4-6 \mathrm{~min} \text { after injection })^{[4,9]}$. Most malignancies within the liver derive their blood supply from the hepatic arteries and not the portal vein. As a result, malignancy typically manifests on CEUS as hyperenhancing compared to the surrounding liver on the arterial phase and as hypoenhancing in the portal venous and late phases. This temporal reduction in the enhancement of a lesion from earlier to later phases is also known as washout ${ }^{[9]}$. It is important to note that the washout of CEUS is different from that of CT or MRI, as microbubble contrast agents of CEUS are purely intravascular, whereas CT and MR contrast agents are both intravascular as well as interstitial. As a result, fibrous lesions such as intrahepatic cholangiocarcinoma (ICC) will appear to have delayed enhancement by CT or MRI, while they will demonstrate washout on CEUS ${ }^{[10]}$.

\section{ADVANTAGES AND LIMITATIONS OF CEUS}

CEUS has a number of advantages as an imaging modality. CEUS is a real-time imaging technique with much higher temporal resolution than CT or MRI, and therefore it does not suffer from mistiming or acquiring contrast phases that are too early or too late, as can be seen with CT or MRI ${ }^{[10]}$. CEUS is highly sensitive to the microbubble contrast agents ${ }^{[7]}$. Therefore, CEUS is a good modality to confirm subtle enhancement when it cannot be seen on CT/MRI ${ }^{[5,11]}$.

CEUS does not use ionizing radiation as does CT. CEUS is also less costly than CT or MRI ${ }^{[12]}$. The microbubble contrast agent for CEUS is safe in patients with severe renal impairment, unlike those used in CT or MRI ${ }^{[13,14]}$. CEUS is approved for the pediatric population and is a very safe alternative that does not require sedation or anesthesia ${ }^{[15]}$.

CEUS has some potential limitations that the practitioner must recognize to tailor its use appropriately. CEUS is usually not suitable for staging of hepatocellular carcinoma, as it is difficult to survey the entire liver effectively with CEUS ${ }^{[16]}$. In general, a few lesions can be characterized in one session of CEUS.

CEUS may require more planning and time on the part of the interpreting physician as lesions of interest must be identified on the precontrast portion of the examination and correlated to the prior imaging ${ }^{[5,17]}$. As a result, the interpreting physician is often involved in the acquisition of CEUS. As CEUS is acquired by a sonographer and/or interpreting physician, it is typically more operator-dependent than CT or MRI. The microbubble imaging agent Lumason ${ }^{\bullet}$ (Bracco Diagnostics, Monroe Township, NJ) became the first agent approved by the US Food and Drug Administration (FDA) for contrast-enhanced abdominal ultrasound imaging in adults and children in April 2016. While this agent has been used worldwide under the name SonoVue ${ }^{\circ}$ for well over a decade before this ${ }^{[13]}$, given the relatively short history of FDA-approved microbubble contrast agents, practitioner knowledge of CEUS acquisition techniques and interpretation is generally less than conventional ultrasound, CT, and MRI.

\section{HISTORY AND BACKGROUND OF CEUS LI-RADS}

The American College of Radiology (ACR) Liver Reporting and Data System (LI-RADS ${ }^{\circ}$ ) was created as a standardized reporting system to facilitate consistent and high-quality technique, interpretation, reporting, 
and data collection for diagnosis of HCC in patients at risk of HCC, initially for CT and MRI in $2011^{[18]}$. Recognizing the value and worldwide utilization of CEUS to diagnose HCC, ACR convened a Working Group of international experts in CEUS to develop CEUS LI-RADS starting in April 2014. The first official version of CEUS LI-RADS (version 2016) was published online in September $2016^{[19]}$.

Similar to CT/MRI LI-RADS ${ }^{\circledR}$, CEUS LI-RADS ${ }^{\circledR}$ is updated at regular intervals. As of the time of writing, the Working Group is finalizing the CEUS LI-RADS ${ }^{\circledR}$ v2019 manual. The most updated versions of LI-RADS ${ }^{\star}$, including CEUS LI-RADS ${ }^{\circ}$ version 2017, can be found on the ACR website (https://www.acr.org/ClinicalResources/Reporting-and-Data-Systems/LI-RADS/).

CEUS is recognized as one of the imaging modalities for HCC diagnosis by many societies worldwide including European Association for the Study of the Liver (EASL) ${ }^{[2]}$, the Asian Pacific Association for the Study of the Liver (APASL) ${ }^{[20]}$, Japanese Society of Hepatology ${ }^{[21]}$, Korean Liver Cancer Study GroupNational Cancer Center ${ }^{[22]}$, Canadian Association for the Study of the Liver (CASL) $)^{[23]}$, Italian Association for the Study of the Liver (AISF) ${ }^{[24]}$, and the World Federation for Ultrasound in Medicine and BiologyEuropean Federation of Society for Ultrasound in Medicine and Biology (WFUMB-EFSUMB) ${ }^{[17]}$. Historically it was removed in the prior American Association for the Study of Liver Diseases (AASLD) guidelines $^{[25]}$ and EASL 2012 guidelines $^{[26]}$ due to the concern of misdiagnosis of ICC as HCC based on a small retrospective study ${ }^{[27]}$. The authors reviewed 21 ICC cases retrospectively and reported 10/21 (47.6\%) of ICC showed homogeneous hyperenhancement followed by a washout on CEUS, therefore they could have been misdiagnosed as HCC. However, subsequent experience and publications do not support the interpretation of this small retrospective study ${ }^{[2-30]}$. While current EASL guidelines now endorse CEUS ${ }^{[2]}$, AASLD has not included CEUS in its new guidelines ${ }^{[3]}$. More data and experience in the United States may help recognition of CEUS for HCC diagnosis in the United States.

\section{MAJOR FEATURES OF CEUS LI-RADS ${ }^{\circledR}$}

The characteristic appearance of HCC by CEUS is due to the purely intravascular nature of the microbubble contrast agent as well as the biology of HCC, which is a vascular tumor that derives its blood supply from the hepatic artery (and not the portal vein). As a result, HCC has a typical CEUS appearance of arterial phase hyperenhancement (APHE) and relative hypoenhancement (washout) in the portal venous or late phase compared to the surrounding liver ${ }^{[9]}$. The washout of HCC is most commonly late (defined as $>60 \mathrm{~s}$ ) and mild. As a result of these observations regarding the appearance of HCC by CEUS, the "major features" that are used to define HCC by CEUS LI-RADS ${ }^{\star}$ are arterial phase hyperenhancement and late and mild washout $^{[19]}$.

\section{DESCRIPTION OF THE CEUS LI-RADS ALGORITHM}

The algorithm of CEUS LI-RADS ${ }^{\bullet}$ integrates the major features of HCC by CEUS (enhancement and washout) as well as size to stratify the likelihood of HCC by imaging appearance ${ }^{[19]}$. The stratification is performed according to the same Likert scale of CT/MRI LI-RADS ${ }^{\circ}$, from LI-RADS 1 (LR-1) meaning definitely benign to LI-RADS 5 (LR-5) meaning definitely HCC [Table 1]. Figure 1 is the CEUS LI-RADS ${ }^{\bullet}$ algorithm and its diagnostic table for LR-3 to LR-5.

If one or more major features cannot be assessed due to image omission or degradation, the nodule/ observation should be designated as LR-NC (non-categorizble).

Tumor in a vein (LR-TIV) is categorized when the classic arterial hyperenhancement and late mild washout of HCC are seen in soft tissue mass within a vein. A review and subsequent meta-analysis of the diagnostic accuracy of CEUS for diagnosis of a tumor in vein support high sensitivity and specificity from several 


\section{Table 1. The CEUS LI-RADS categories}

\begin{tabular}{|c|c|}
\hline CEUS LR-NC & $\begin{array}{l}\text { Not Categorizable: } \\
\text { The observation that cannot be meaningfully categorized because image omission or degradation prevents the } \\
\text { assessment of one or more major features }\end{array}$ \\
\hline CEUS LR-1 & $\begin{array}{l}\text { Definitely Benign: } \\
100 \% \text { certainty that the observation is not malignant }\end{array}$ \\
\hline CEUS LR-2 & $\begin{array}{l}\text { Probably Benign: } \\
\text { High probability but not } 100 \% \text { certainty that the observation is not malignant }\end{array}$ \\
\hline CEUS LR-3 & $\begin{array}{l}\text { Intermediate probability of malignancy: } \\
\text { Non-malignant and malignant entities each have a moderate probability }\end{array}$ \\
\hline CEUS LR-4 & $\begin{array}{l}\text { Probably HCC: } \\
\text { High probability but not } 100 \% \text { certainty that the observation is HCC }\end{array}$ \\
\hline CEUS LR-5 & $\begin{array}{l}\text { Definitely HCC: } \\
100 \% \text { certainty that the observation is HCC }\end{array}$ \\
\hline CEUS LR-M & $\begin{array}{l}\text { Probably or definitely malignant, not HCC specific: } \\
\text { High probability or } 100 \% \text { certainty observation is malignant but features are not HCC specific }\end{array}$ \\
\hline CEUS LR-TIV & $\begin{array}{l}\text { Tumor in vein: } \\
100 \% \text { certainty there is malignancy with tumor in vein }\end{array}$ \\
\hline
\end{tabular}

CEUS LI-RADS: Contrast-Enhanced Ultrasound Liver Reporting and Data System; HCC: hepatocellular carcinoma

Untreated observation visible on precontrast US and without pathologic proof
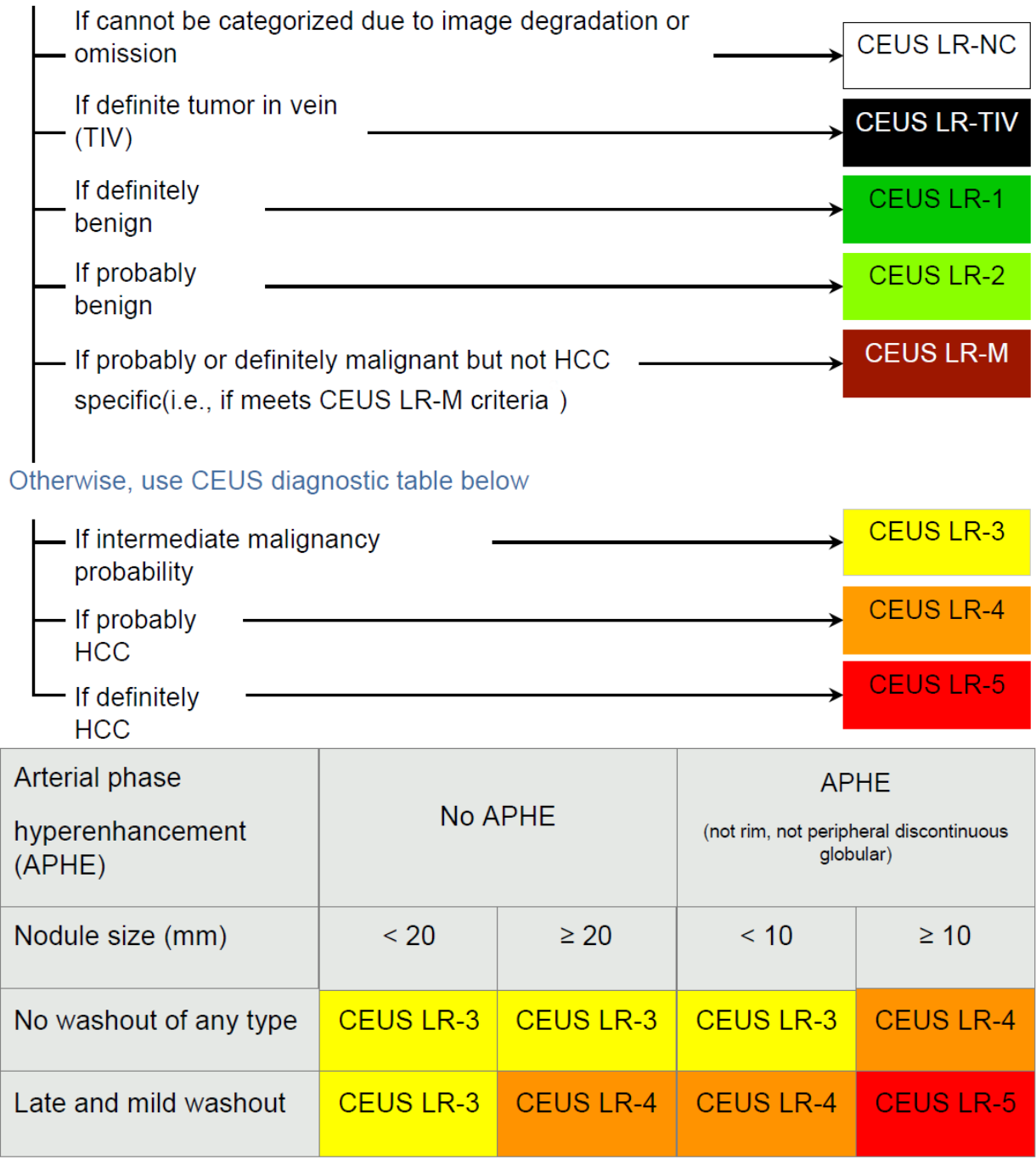

Figure 1. The CEUS LI-RADS Algorithm and Diagnostic Table (reproduced with permission from the ACR). CEUS LI-RADS: ContrastEnhanced Ultrasound Liver Reporting and Data System; HCC: hepatocellular carcinoma; ACR: American College of Radiology 
Grayscale ultrasound

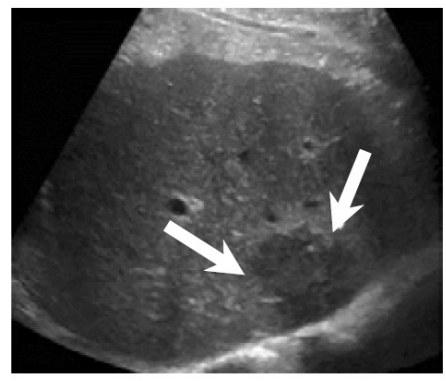

Hypoechoic mass
Arterial phase (16 sec)

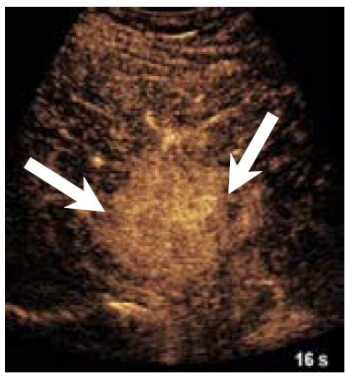

APHE
Arterial phase

(34 sec)

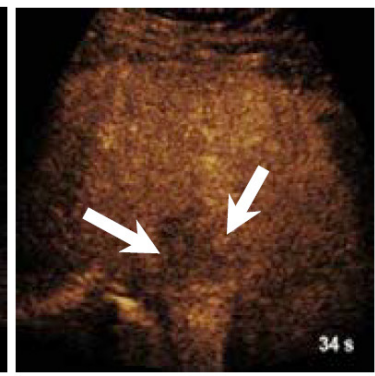

Rapid

washout
Late phase

(4 min)

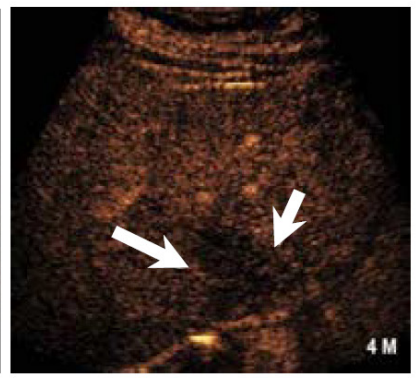

washout

Figure 2. Example case of a LR-M nodule. 78-year-old male with HBV and a growing nodule on MRI, approximately $3 \mathrm{~cm}$, previously diagnosed as a hemangioma on MRI. CEUS imaging demonstrated arterial phase hyperenhancement at $16 \mathrm{~s}$ followed by almost immediate washout by $34 \mathrm{~s}$. Biopsy showed the mass to be intrahepatic cholangiocarcinoma (image reproduced with permission from the ACR). MRI: magnetic resonance imaging; HBV: hepatitis B virus; CEUS: contrast-enhanced ultrasound; ACR: American College of Radiology

published studies ${ }^{[31,32]}$, with a pooled sensitivity of $94 \%$ and a pooled specificity of $99 \%$. On the other hand, diagnosis using CT or MRI can be more challenging with a poorer reported performance for more widely available CT/MRI techniques. The reported sensitivity and specificity for contrast-enhanced CT is $43 \%$ and $100 \%$, respectively ${ }^{[33]}$. More recent reports of conventional MRI findings suggest that sensitivity and specificity up to $100 \%$ and $90 \%$, respectively, can be achieved with a "careful" evaluation of findings ${ }^{[34]}$. Arguably, diagnosis is simpler and more direct using CEUS as indirect signs (such as primary mass size and distance from a vein) are not used.

LR-1 (definitely benign) observations include cysts, hemangiomas, focal hepatic fat deposition, focal hepatic fat sparing, and hypertrophic pseudo mass. The reader is referred to several excellent reviews for a thorough description of the imaging appearance of these entities ${ }^{[35-39]}$. An examination performed as a followup of observation could also be categorized as LR-1 if the observation spontaneously disappeared on followup.

LR-2 (probably benign) observations include several classes of nodules. First, a distinct nodule was seen on non-contrast grayscale ultrasound $<10 \mathrm{~mm}$ without APHE is categorized at LR-2 (if $\geq 10 \mathrm{~mm}$, the nodule would be categorized as LR-3). Nonmasslike (i.e., regional or geographic) isoenhancement of any size would also be categorized as LR-2. Second, an LR-3 nodule that has been stable for $\geq 2$ years could be downgraded to an LR-2. Third, observations that are probably LR-1 - cysts, hemangiomas, focal hepatic fat deposition, focal hepatic fat sparing, and hypertrophic pseudo mass - but are not definite by imaging criteria would be categorized as LR-2.

LR-M (Probably or malignant, not HCC specific) is an important category. Major criteria include rim arterial phase hyperenhancement with any washout, OR early $(<1 \mathrm{~min})$ OR marked (becomes black, punched out appearance within $2 \mathrm{~min}$ ). An example LR-M case is shown in Figure 2. The existence of the LR-M category is crucial to maintain the high specificity of LR-5. All LR-5 should be HCC, while not all HCC are categorized in LR-5. LR-M are typically non-hepatocellular malignancy such as ICC, and metastasis, however, some HCC especially poorly differentiated HCC is most likely categorized as LR-M. LR-M are recommended to have a biopsy for diagnosis, therefore, the diagnosis will be accurately achieved not by imaging but by histology. 


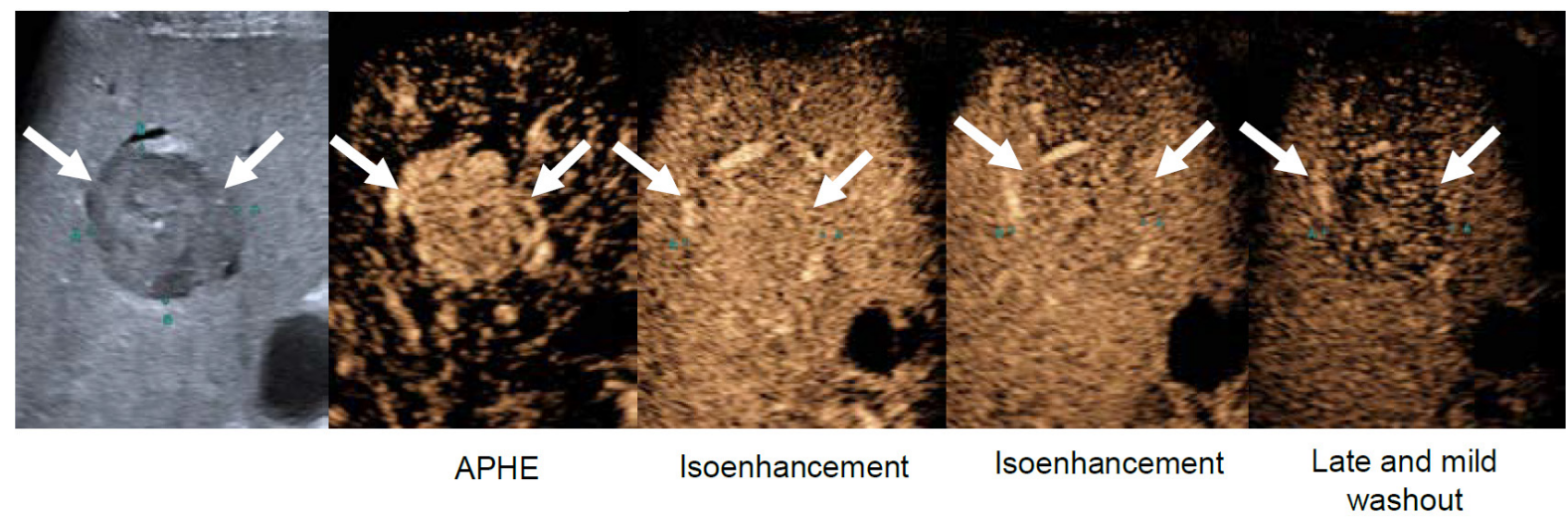

Figure 3. Example case of a LR-5 nodule. A $34 \mathrm{~mm}$ observation in a 66 year-old man with HCV cirrhosis, demonstrating arterial phase hyperenhancement (APHE), isoenhancement through the portal venous and late phase, with late mild washout observed at 5 min after contrast injection. (Image reproduced with permission from the ACR). HCV: hepatitis C virus; ACR: American College of Radiology

After the above lesions are categorized appropriately and therefore excluded from consideration as possible or definite HCC, the CEUS LI-RADS ${ }^{\bullet}$ diagnostic table is applied to categorize LR-3, LR-4, and LR-5. The table integrates the presence of arterial hyperenhancement, presence of late mild washout, and nodule size [Figure 1]. It is important to distinguish that the arterial hyperenhancement used to categorize nodules as LR-3, LR-4, or LR-5 should not be "rim" or peripherally continuous, as this is not characteristic of HCC. Rather, such arterial phase hyperenhancement would categorize a nodule as LR-M (probably or definitely malignant but not HCC specific). Also, if a nodule shows washout and it is not the late mild washout typical of HCC, then such a nodule would be categorized as LR-M. Examples of washout atypical of HCC include early washout (within $60 \mathrm{~s}$ ) and marked washout that results in a "punched out" appearance within 2 min. Figure 3 is a typical HCC (LR-5) case.

After the above algorithm is applied, ancillary imaging features may be used to upgrade or downgrade a nodule between CEUS LI-RADS ${ }^{\circledR}$ categories. These ancillary imaging features include nodule-in-nodule/ mosaic architecture (favoring HCC in particular), definite growth (favoring malignancy, not HCC in particular), size stability more than 2 years and size reduction (favoring benignity). Of note, a nodule cannot be upgraded to LR-5 in keeping with stringent criteria to maintain high specificity of an LR-5 categorization, and imaging features can only upgrade/downgrade by a maximum of one category (i.e., LR-3 to LR-4). Additionally, CEUS LI-RADS ${ }^{\circledR}$ has fewer ancillary imaging features than CT/MR LI-RADS .

\section{INDICATIONS FOR CEUS}

Taking into account the advantages and potential drawbacks of CEUS, as well as the major features and algorithm of CEUS LI-RADS ${ }^{\oplus}$, the following are common indications for CEUS in patients at risk for HCC: (1) assessment of nodules $\geq 10 \mathrm{~mm}$ detected on surveillance ultrasound; (2) assessment of observations that are indeterminate on prior CT or MRI (i.e., LR-3, LR-4, or LR-M); (3) detection of arterial phase hyperenhancement when it is suspected that contrast mistiming is suspected as a cause of lack of arterial enhancement on prior CT or MRI; (4) detection of CEUS washout when CT or MRI washout is indeterminate but shows APHE; (5) further evaluation of biopsied observation with inconclusive histology; (6) guiding biopsy or percutaneous ablation of observations difficult to visualize with precontrast US; (7) guiding biopsy of heterogeneous observations; (8) monitor changes in enhancement pattern over time for selected CEUS LR-3 or CEUS LR-4 observations; and (9) differentiating tumor in vein ('tumor thrombus') from bland thrombus. With regards to the first indication listed above, studies and experience have shown 
that having CEUS available at the time of surveillance ultrasound reduces time to diagnosis, healthcare expenditures, and patient anxiety ${ }^{[12,40]}$.

Similar to CT/MRI LI-RADS ${ }^{\star}$, CEUS LI-RADS ${ }^{\star}$ should only be applied in populations at risk for HCC by AASLD guidelines, including patients with cirrhosis of any etiology or noncirrhotic patients with chronic hepatitis B.

\section{DIFFERENCES BETWEEN CEUS LI-RADS AND CT/MRI LI-RADS}

As the reader may be more acquainted with CT/MRI LI-RADS ${ }^{\circledR}$, we will highlight some differences between $\mathrm{CEUS} \mathrm{LI-RADS}^{\circ}$ and CT/MRI LI-RADS ${ }^{\circ}$ to reinforce the utility and some unique aspects of CEUS.

CEUS practitioners have long used the term "nodule" to describe possible lesions of interest, so in CEUS LI-RADS "nodule" is often used interchangeably with the term "observation". In CT/MRI LI-RADS", the term "observation" is favored as APHE on CT/MRI may be due to arterioportal shunting or other pseudolesions. By contrast, CEUS does not show these AP shunts and is a very important tool to differentiate them from HCC without washout ${ }^{[10]}$. Conversely, a suspected arterioportal shunt on CT/MRI associated with a distinct nodule on US will raise suspicion for $\mathrm{HCC}^{[41]}$.

CEUS and CT/MRI exhibit differences in enhancement and washout due to differences in the contrast agents employed for each. CEUS agents are several microns in diameter, which confines them to be purely intravascular contrast agents. CT/MRI contrast agents are much smaller in size and therefore have an extracellular interstitial phase of contrast. As a result of these differences, a "capsule" appearance is typically seen in HCC when assessed by CT/MRI and is therefore a major criterion for categorization as LR-5 by CT/MRI LI-RADS ${ }^{\circ}$. Capsule appearance is not seen by CEUS and is therefore not one of the major features used for categorization by CEUS LI-RADS ${ }^{\text {[10] }}$.

\section{DISCUSSION}

Structured reporting systems such as BI-RADS ${ }^{\circledR}$ and LI-RADS ${ }^{\star}$ emerged due to the need for increased consistency of imaging diagnostics ${ }^{[18]}$. These systems typically are created based on existing data and expertise, but the validation of real-world use is critical. An international multi-center prospective clinical trial has been organized by members of the CEUS LI-RADS ${ }^{\circledR}$ working group to assess the diagnostic accuracy of CEUS for HCC diagnosis in patients at risk for HCC, assess the interreader reliability of diagnosis, and to validate CEUS LI-RADS ${ }^{[42]}$. Recruitment is still ongoing as of the time of writing.

There are existing retrospective data to support the use of CEUS LI-RADS ${ }^{\circledR}$ and provide the prevalence of HCC within each of the categories from a large international case series out of Europe and Canada. Terzi et al. ${ }^{[43]}$ showed that in 848 patients with 1006 lesions of median size $2 \mathrm{~cm}$, the specificity of LR- 5 for HCC diagnosis was $98.5 \%$. Concern has been raised historically that CEUS may misdiagnose ICC, however, this was not found to be of concern in this large case series. None of the 519 LR-5 nodules were pure ICC, although one nodule was found to represent mixed HCC-ICC. Regarding the LR-M category ( $8 \%$ of lesions), $38 \%$ were ICC, $10 \%$ were mixed HCC-ICC, $48 \%$ were HCC, and $2 \%$ were metastases. A large study of 2020 patients with hepatitis B virus (HBV) infection from China validated CEUS LI-RADS ${ }^{\circ}$ for patient populations in whom HBV is endemic - with LR-5 showing high specificity of $96 \%$ and positive predictive value of $98 \%$ for the diagnosis of $\mathrm{HCC}^{[4]}$. More recently, similar diagnostic accuracy was observed in another large retrospective study of patients from China who were predominantly (92\%) HBV positive with LR-5 showing a sensitivity and specificity of $73 \%$ and $97 \%$, respectively for nodules $20 \mathrm{~mm}$ or less ${ }^{[45]}$.

Retrospective data regarding the interreader reliability of CEUS LI-RADS ${ }^{\bullet}$ is encouraging. The largest 
retrospective study of 1,366 patients showed excellent specificity for HCC of $90.2 \%$ with an interreader agreement of 0.61-0.73 for LI-RADS categories. Another retrospective study of 258 patients comparing inexperienced and experienced radiologists showed that not only was interreader agreement high between the two groups, but diagnostic accuracy was also excellent when using CEUS LI-RADS (sensitivity 84.2\%$87.5 \%$, sensitivity $90.6 \%-97 \%)^{[46]}$. A smaller case series of 50 patients found that the interreader agreement of CEUS LI-RADS categories was only fair, with a kappa of 0.309, and that agreement of APHE was higher than that of washout ${ }^{[47]}$.

\section{CONCLUSION}

In summary, CEUS LI-RADS ${ }^{\oplus}$ is a recent addition to the constellation of LI-RADS ${ }^{ø}$ structured reporting systems for HCC. Retrospective data supports a high specificity for the diagnosis of HCC by the LR- 5 category, and a large multi-center prospective validation study is ongoing. Additional future areas of expansion of CEUS LI-RADS ${ }^{\oplus}$ include treatment assessment.

\section{DECLARATIONS}

\section{Author contributions}

Conception and writing of this work: Vezeridis AM, Kono Y

\section{Availability of data and materials}

Not applicable.

\section{Financial support and sponsorship}

None.

\section{Conflicts of interest}

Both authors declared that there are no conflicts of interest.

\section{Ethical approval and consent to participate}

Not applicable.

\section{Consent for publication}

Not applicable.

\section{Copyright}

(c) The Author(s) 2020

\section{REFERENCES}

1. Heimbach JK, Kulik LM, Finn RS, Sirlin CB, Abecassis MM, et al. AASLD guidelines for the treatment of hepatocellular carcinoma. Hepatology 2018;67:358-80.

2. European Association for the Study of the Liver. Electronic address eee, European Association for the Study of the L. EASL Clinical Practice Guidelines: management of hepatocellular carcinoma. J Hepatol 2018;69:182-236.

3. Marrero JA, Kulik LM, Sirlin CB, Zhu AX, Finn RS, et al. Diagnosis, staging, and management of hepatocellular carcinoma: 2018 Practice Guidance by the American Association for the Study of Liver Diseases. Hepatology 2018;68:723-50.

4. Claudon M, Dietrich CF, Choi BI, Cosgrove DO, Kudo M, et al. Guidelines and good clinical practice recommendations for contrast enhanced ultrasound (CEUS) in the liver - update 2012: a WFUMB-EFSUMB initiative in cooperation with representatives of AFSUMB, AIUM, ASUM, FLAUS and ICUS. Ultrasound Med Biol 2013;39:187-210.

5. Jo PC, Jang HJ, Burns PN, Burak KW, Kim TK, et al. Integration of contrast-enhanced US into a multimodality approach to imaging of nodules in a cirrhotic liver: how i do it. Radiology 2017;282:317-31.

6. Quaia E. Microbubble ultrasound contrast agents: an update. Eur Radiol 2007;17:1995-2008.

7. Ophir J, Parker KJ. Contrast agents in diagnostic ultrasound. Ultrasound Med Biol 1989;15:319-33.

8. Wilson SR, Burns PN. An algorithm for the diagnosis of focal liver masses using microbubble contrast-enhanced pulse-inversion 
sonography. AJR Am J Roentgenol 2006;186:1401-12.

9. Yang HK, Burns PN, Jang HJ, Kono Y, Khalili K, et al. Contrast-enhanced ultrasound approach to the diagnosis of focal liver lesions: the importance of washout. Ultrasonography 2019;38:289-301.

10. Kim TK, Noh SY, Wilson SR, Kono Y, Piscaglia F, et al. Contrast-enhanced ultrasound (CEUS) liver imaging reporting and data system (LI-RADS) 2017 - a review of important differences compared to the CT/MRI system. Clin Mol Hepatol 2017;23:280-9.

11. Sugimoto K, Moriyasu F, Shiraishi J, Saito K, Taira J, et al. Assessment of arterial hypervascularity of hepatocellular carcinoma: comparison of contrast-enhanced US and gadoxetate disodium-enhanced MR imaging. Eur Radiol 2012;22:1205-13.

12. Streb JW, Tchelepi H, Malhi H, Deurdulian C, Grant EG. Retrospective analysis of contrast-enhanced ultrasonography effectiveness in reducing time to diagnosis and imaging-related expenditures at a single large United States County hospital. Ultrasound Q 2019;35:99-102.

13. Piscaglia F, Bolondi L, Italian Society for Ultrasound in M, Biology Study Group on Ultrasound Contrast A. The safety of Sonovue in abdominal applications: retrospective analysis of 23188 investigations. Ultrasound Med Biol 2006;32:1369-75.

14. Yusuf GT, Sellars ME, Deganello A, Cosgrove DO, Sidhu PS. Retrospective analysis of the safety and cost implications of pediatric contrast-enhanced ultrasound at a single center. AJR Am J Roentgenol 2017;208:446-52.

15. Ntoulia A, Anupindi SA, Darge K, Back SJ. Applications of contrast-enhanced ultrasound in the pediatric abdomen. Abdom Radiol (NY) 2018;43:948-59.

16. Lyshchik A, Kono Y, Dietrich CF, Jang HJ, Kim TK, et al. Contrast-enhanced ultrasound of the liver: technical and lexicon recommendations from the ACR CEUS LI-RADS working group. Abdom Radiol (NY) 2018;43:861-79.

17. Claudon M, Dietrich CF, Choi BI, Cosgrove DO, Kudo M, et al. Guidelines and good clinical practice recommendations for Contrast Enhanced Ultrasound (CEUS) in the liver - update 2012: a WFUMB-EFSUMB initiative in cooperation with representatives of AFSUMB, AIUM, ASUM, FLAUS and ICUS. Ultrasound Med Biol 2013;39:187-210.

18. Tang A, Singal AG, Mitchell DG, Hecht EM, Fowler KJ, et al. Introduction to the Liver Imaging Reporting and Data System for Hepatocellular Carcinoma. Clin Gastroenterol Hepatol 2019;17:1228-38.

19. Kono Y, Lyshchik A, Cosgrove D, Dietrich CF, Jang HJ, et al. Contrast Enhanced Ultrasound (CEUS) Liver Imaging Reporting and Data System (LI-RADS ${ }^{\circledR}$ ): the official version by the American College of Radiology (ACR). Ultraschall Med 2017;38:85-6.

20. Omata M, Cheng AL, Kokudo N, Kudo M, Lee JM, et al. Asia-Pacific clinical practice guidelines on the management of hepatocellular carcinoma: a 2017 update. Hepatol Int 2017;11:317-70.

21. Kudo M, Matsui O, Izumi N, Iijima H, Kadoya M, et al. JSH Consensus-Based Clinical Practice Guidelines for the Management of Hepatocellular Carcinoma: 2014 Update by the Liver Cancer Study Group of Japan. Liver Cancer 2014;3:458-68.

22. Korean Liver Cancer Study G, National Cancer Center K. 2014 Korean Liver Cancer Study Group-National Cancer Center Korea practice guideline for the management of hepatocellular carcinoma. Korean J Radiol 2015;16:465-522.

23. Burak KW, Sherman M. Hepatocellular carcinoma: consensus, controversies and future directions. A report from the Canadian Association for the Study of the Liver Hepatocellular Carcinoma Meeting. Can J Gastroenterol Hepatol 2015;29:178-84.

24. Italian Association for the Study of the L, Panel AE, Committee AC, Bolondi L, Cillo U, Colombo M, et al. Position paper of the Italian Association for the Study of the Liver (AISF): the multidisciplinary clinical approach to hepatocellular carcinoma. Dig Liver Dis 2013;45:712-23.

25. Bruix J, Sherman M, American Association for the Study of Liver D. Management of hepatocellular carcinoma: an update. Hepatology 2011;53:1020-2.

26. EASL-EORTC clinical practice guidelines: management of hepatocellular carcinoma. J Hepatol 2012;56:908-43.

27. Vilana R, Forner A, Bianchi L, Garcia-Criado A, Rimola J, et al. Intrahepatic peripheral cholangiocarcinoma in cirrhosis patients may display a vascular pattern similar to hepatocellular carcinoma on contrast-enhanced ultrasound. Hepatology 2010;51:2020-9.

28. Han J, Liu Y, Han F, Li Q, Yan C, et al. The degree of contrast washout on contrast-enhanced ultrasound in distinguishing intrahepatic cholangiocarcinoma from hepatocellular carcinoma. Ultrasound Med Biol 2015;41:3088-95.

29. Li R, Yuan MX, Ma KS, Li XW, Tang CL, et al. Detailed analysis of temporal features on contrast enhanced ultrasound may help differentiate intrahepatic cholangiocarcinoma from hepatocellular carcinoma in cirrhosis. PLoS One 2014;9:e98612.

30. Wildner D, Bernatik T, Greis C, Seitz K, Neurath MF, et al. CEUS in hepatocellular carcinoma and intrahepatic cholangiocellular carcinoma in 320 patients - early or late washout matters: a subanalysis of the DEGUM multicenter trial. Ultraschall Med 2015;36:132-9.

31. Danila M, Sporea I, Popescu A, Șirli R. Portal vein thrombosis in liver cirrhosis - the added value of contrast enhanced ultrasonography. Med Ultrason 2016;18:218-33.

32. Chen J, Zhu J, Zhang C, Song Y, Huang P. Contrast-enhanced ultrasound for the characterization of portal vein thrombosis vs tumor-invein in HCC patients: a systematic review and meta-analysis. Eur Radiol 2020;30:2871-80.

33. Tublin ME, Dodd GD, Baron RL. Benign and malignant portal vein thrombosis: differentiation by CT characteristics. AJR Am J Roentgenol 1997;168:719-23.

34. Sandrasegaran K, Tahir B, Nutakki K, Akisik FM, Bodanapally U, et al. Usefulness of conventional MRI sequences and diffusionweighted imaging in differentiating malignant from benign portal vein thrombus in cirrhotic patients. AJR Am J Roentgenol 2013;201:1211-9.

35. Burrowes DP, Medellin A, Harris AC, Milot L, Wilson SR. Contrast-enhanced US approach to the diagnosis of focal liver masses. Radiographics 2017;37:1388-400.

36. Burrowes DP, Kono Y, Medellin A, Wilson SR. Update: contrast-enhanced US approach to the diagnosis of focal liver masses. Radiographics 2020;40:E16-20.

37. Dietrich CF, Tana C, Caraiani C, Dong Y. Contrast enhanced ultrasound (CEUS) imaging of solid benign focal liver lesions. Expert Rev 
Gastroenterol Hepatol 2018;12:479-89.

38. Dietrich CF. Contrast-enhanced ultrasound of benign focal liver lesions. Ultraschall Med 2019;40:12-29.

39. Kim TK, Lee E, Jang HJ. Imaging findings of mimickers of hepatocellular carcinoma. Clin Mol Hepatol 2015;21:326-43.

40. Lanka B, Jang HJ, Kim TK, Burns PN, Wilson SR. Impact of contrast-enhanced ultrasonography in a tertiary clinical practice. J Ultrasound Med 2007;26:1703-14.

41. Hu J, Bhayana D, Burak KW, Wilson SR. Resolution of indeterminate MRI with CEUS in patients at high risk for hepatocellular carcinoma. Abdom Radiol (NY) 2020;45:123-33.

42. Kono Y, Sirlin CB, Fetzer DT, Kim TK, Rodgers SK, et al. Time to clarify common misconceptions about the liver imaging reporting and data system for contrast-enhanced US. Radiology 2020;295:245-7.

43. Terzi E, Iavarone M, Pompili M, Veronese L, Cabibbo G, et al. Contrast ultrasound LI-RADS LR-5 identifies hepatocellular carcinoma in cirrhosis in a multicenter restropective study of 1,006 nodules. J Hepatol 2018;68:485-92.

44. Zheng W, Li Q, Zou XB, Wang JW, Han F, et al. Evaluation of contrast-enhanced US LI-RADS version 2017: application on 2020 liver nodules in patients with hepatitis B infection. Radiology 2020;294:299-307.

45. Huang JY, Li JW, Lu Q, Luo Y, Lin L, et al. Diagnostic accuracy of CEUS LI-RADS for the characterization of liver nodules $20 \mathrm{~mm}$ or smaller in patients at risk for hepatocellular carcinoma. Radiology 2020;294:329-39.

46. Wang JY, Feng SY, Xu JW, Li J, Chu L, et al. Usefulness of the contrast-enhanced ultrasound liver imaging reporting and data system in diagnosing focal liver lesions by inexperienced radiologists. J Ultrasound Med 2020; doi: 10.1002/jum.15242.

47. Schellhaas B, Hammon M, Strobel D, Pfeifer L, Kielisch C, et al. Interobserver and intermodality agreement of standardized algorithms for non-invasive diagnosis of hepatocellular carcinoma in high-risk patients: CEUS-LI-RADS versus MRI-LI-RADS. Eur Radiol 2018;28:4254-64. 